\title{
NOUVELLE
}

\section{Communication foie/tube digestif}

Rôle du microbiote dans la carcinogenèse hépatique

Jamila Faivre ${ }^{1,2,3}$, Christian Bréchot ${ }^{1,2}$, Nicolas Moniaux ${ }^{1,2}$
${ }^{1}$ Inserm U785, centre hépatobiliaire,

12-14, avenue Paul Vaillant Couturier, 94800 Villejuif, France ;

${ }^{2}$ Université Paris-Sud, Faculté de médecine,

94800 Villejuif, France ;

${ }^{3}$ Département d'hématologie et de biologie des tumeurs, Hôpital Paul Brousse, 94800 Villejuif, France.

nicolas.moniaux@inserm.fr
Immunité innée et défense de l'hôte contre les pathogènes

Au cours des cinq dernières années, les technologies les plus récentes de séquençage à haut débit et de métagénomique ont révélé des déséquilibres de la flore intestinale associés à des pathologies de «société », comme les maladies immuno-allergiques, métaboliques ou dégénératives. Des maladies aussi diverses que le diabète [11], l'obésité, les maladies inflammatoires chroniques de l'intestin [12], les maladies cardiovasculaires, les maladies hépatiques, l'asthme, l'eczéma et même l'autisme, ont été associées à des perturbations quantitatives et qualitatives du microbiote intestinal (dysbiose).

Concernant les maladies du foie, des études récentes ont suggéré que le microbiote intestinal était impliqué dans la progression de la stéatose hépatique non alcoolique (NAFLD, non alcoholic fatty liver disease) et jouerait un rôle promoteur dans le développement du carcinome hépatocellulaire $(\mathrm{CHC})$. Le rôle récemment évoqué et largement controversé dans I'hépatocarcinogenèse du système LPS/ TLR4 (lipopolysaccharide/récepteur Tolllike 4) et, plus généralement, des motifs bactériens intestinaux et des récepteurs cellulaires (de type PRR, pattern recognition receptors) qui leur sont associés, est discuté dans cette revue.

Parmi les PRR, les récepteurs Toll-like (TLR) sont essentiels pour la défense contre les microorganismes [13]. Ils sont capables de déclencher les processus d'immunité innée et d'inflammation en réponse à des signaux de danger de nature procaryote ou eucaryote. Ils reconnaissent des MAMP (microbesassociated molecular pattern) provenant de microorganismes, et des DAMP (danger-associated molecular patterns) provenant de tissus endommagés $(\rightarrow)$ [14]. Après stimulation, ils induisent une

$(\rightarrow)$ Voir la synthèse de Y. Jamilloux et T. Henry, page 975 de ce numéro sécrétion de cytokines pro-inflammatoires, telles que le TNF $\alpha$ (tumor necrosis factor $\alpha$ ) et I'IL-6 (interleukine 6) via une cascade d'activation moléculaire intracellulaire impliquant, entre autres, Myd88 (myeloid differentiation primary response 88) et IRAK (IL1-receptorassociated serine kinase), qui mène à l'activation des facteurs de transcription $N F \kappa B$ et APl (activator protein 1), ainsi qu'à l'activation de facteurs déclenchant la réponse immunitaire adaptative.

Rôle de l'intégrité de la barrière intestinale dans le carcinome hépatocellulaire

Le carcinome hépatocellulaire ( $\mathrm{CHC}$ ) se classe au cinquième rang des cancers et représente la troisième cause de mortalité par cancer dans le monde. II s'agit d'une tumeur génétiquement hétérogène développée dans $85 \%$ des cas sur une cirrhose, le plus souvent d'origine alcoolique ou virale. La répétition d'épisodes inflammatoires, dont l'origine peut être virale (virus des hépatites $B$ et $C$ ), alcoolique et métabolique, et le développement d'hépatopathies chroniques associées, telles que la stéatohépatite (NASH) et la cirrhose, sont des causes majeures de carcinogenèse hépatique. Les cycles successifs d'agres- sion du foie qui précèdent la survenue du CHC conduisent à un véritable remodelage de l'organe (nodules de régénération hépatocytaire). Ce dernier est associé, sur le plan moléculaire, à de profondes altérations des voies de signalisation régulant la prolifération, la mort cellulaire, la migration/invasion cellulaires et l'angiogenèse [1]. Il est, par ailleurs, bien connu que la cirrhose s'accompagne fréquemment d'une altération de l'intégrité de la barrière intestinale associée à une sensibilité accrue des patients cirrhotiques aux infections bactériennes issues du tube digestif. La translocation de produits bactériens, comme le lipopolysaccharide (LPS, composant de la paroi des bactéries Gram négatif), par le système veineux portal contribue ainsi à la pérennisation des lésions inflammatoires du foie $[2,6]$. S’il a été montré que la composition du microbiote de patients cirrhotiques était différente de celle de sujets sains, avec notamment une sur-représentation de bactéries Gram négatif, il est impossible de distinguer la cause de la conséquence.

\section{Implication de la voie LPS/TLR4} dans la carcinogenèse hépatique Une étude pionnière publiée en 2010 a démontré que l'activation de la voie LPS/ TLR4 favorisait la prolifération hépatocytaire via la sécrétion de TNF $\alpha$ et d'IL-6 par les cellules de Kupffer (macrophages résidents du foie), et augmentait la résistance des hépatocytes au stress oxydatif et à l'apoptose avec pour conséquence le développement accéléré de CHC dans un modèle expérimental chimio-induit par la diéthylnitrosamine (DEN) [3]. Dans le 
3

Inflammation augmentée Développement du carcinome hépatocellulaire?

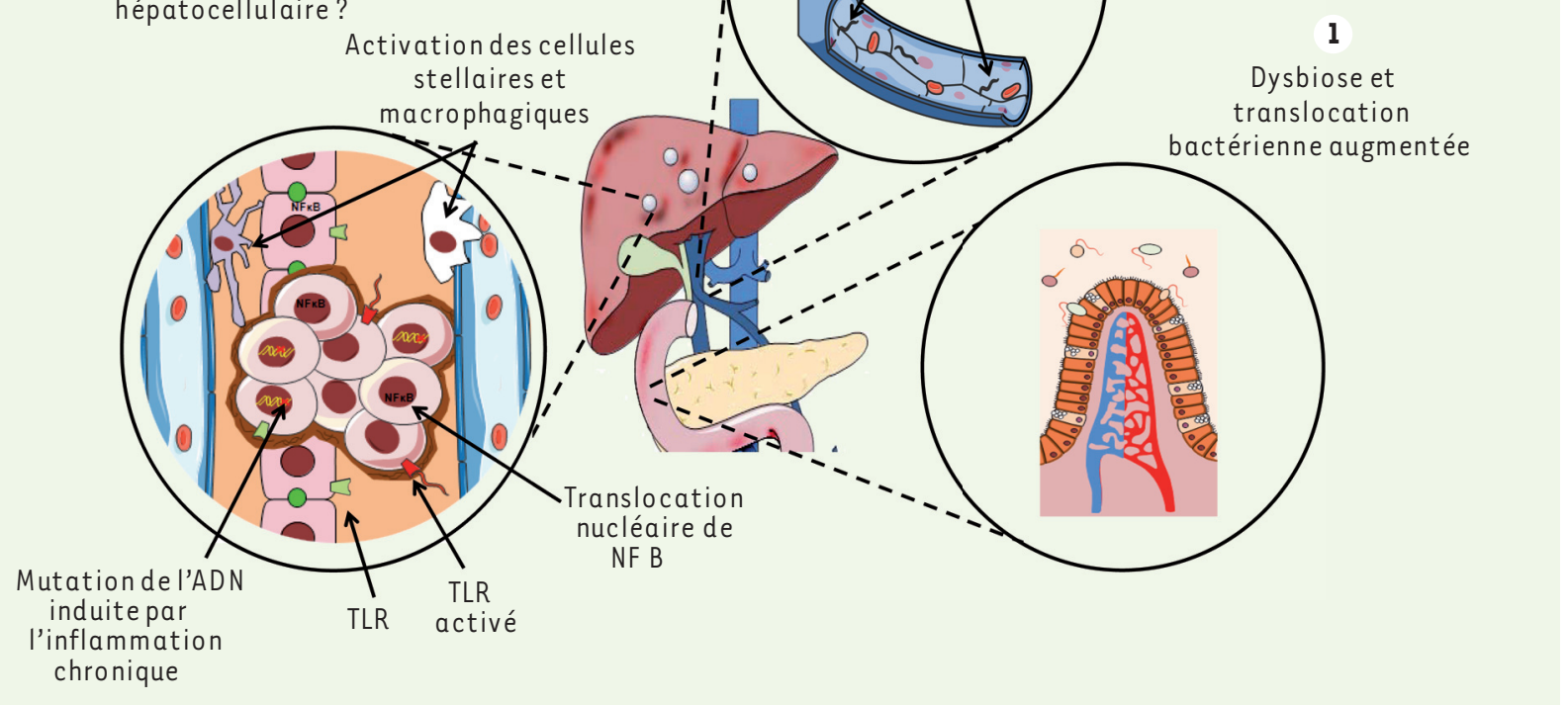

Circulation

entérohépatique de PAMP et/ou de métabolites bactériens 
maintien de la sénescence cellulaire, des flux d'autophagie et de l'expression des protéines de réparation de I'ADN qui, ensemble, formeraient une barrière capable de lutter contre la carcinogenèse hépatique [5].

\section{Méthodologie expérimentale et variabilité du rôle de la voie LPS/TLR4 dans l'hépatocarcinogenèse}

Nombre de résultats contradictoires rapportés dans la littérature sont liés aux différences notables des méthodologies expérimentales employées, à leur diversité et à leur influence variable (et méconnue) sur la composition de l'écosystème intestinal et, par là, sur la réponse inflammatoire et la carcinogenèse. La liste est longue : elle comprend, entre autres, les conditions d'hébergement et de soins des modèles animaux, le type de carcinogenèse induite (dose, schéma d'administration, âge des animaux, hépatopathie chronique sousjacente associée ou non), les caractéristiques de la dysbiose associée qui est elle-même influencée par les procédés de collecte des fèces et d'extraction de I'ADN génomique bactérien, sans oublier l'impact sur la réponse immunoinflammatoire du portage chronique en différents écosystèmes (notamment microbiome et virome) intestinaux et extra-intestinaux. II a également été observé que certains produits antibiotiques ou génotoxiques altéraient sensiblement la composition du microbiote intestinal et semblaient stimuler le développement d'un CHC chimio-induit chez le rat [6]. Dans cette étude, un traitement à base de pénicilline modifiait la composition de la flore commensale murine avec une disparition des bactéries commensales de type Bifidobacterium et Lactobacillus, et une sur-représentation des bactéries Gram négatif de type Escherichia coli et Atopobium, sources principales de LPS circulant [6]. Un tel profil de dysbiose associé à une augmentation du LPS circulant était également retrouvé chez des rats intoxiqués par le DEN n'ayant pas reçu d'antibiothérapie [6]. Ces résultats ne représentent pas une preuve formelle mécanistique reliant un type de microbiote intestinal particulier à la carcinogenèse hépatique. Ils indiquent cependant la nécessité d'analyser les profils métagénomiques bactériens des patients atteints de carcinome hépatocellulaire (ce qui reste à faire) et soulignent l'importance de sélectionner rigoureusement les cohortes de patients cancéreux à étudier et les groupes contrôles. En effet, si la composition bactérienne est directement associée au patrimoine génétique de l'hôte [7], les influences environnementale, nutritionnelle et métabolique semblent être prépondérantes dans la modulation du métagénome intestinal.

Sur le plan clinique, des données expérimentales suggèrent le rôle primordial de la voie LPS/TLR4 dans la carcinogenèse hépatique. Les individus portant une mutation du gène TLR4, polymorphisme rs2149356 T, ont un moindre risque de développer un $\mathrm{CHC}$ secondaire à une infection par le virus de l'hépatite C [9]. De même, chez les patients porteurs d'un CHC, le niveau d'expression de la protéine adaptatrice de la voie des TLR, Myd88, est élevé, et cette expression est directement corrélée à l'agressivité tumorale [10]. Au total, il est bien sûr prématuré de conclure avec certitude au rôle de la voie LPS/TLR dans la promotion du CHC, voie complexe qui se situe à l'interface des mécanismes de défense antimicrobienne, de réparation/ régénération tissulaire, d'orientation de la réponse immunitaire tolérogène ou non tolérogène, et enfin de la carcinogenèse (Figure 1). Cet équilibre est dépendant du patrimoine génétique de chaque individu, de la composition de sa flore commensale, et de la nature et chronicité de l'agression à l'origine de la maladie inflammatoire. Des travaux de recherche expérimentale et clinique sont à l'évidence nécessaires pour mieux nous éclairer sur le rôle des MAMP/TLR dans la physiopathologie hépatique. En outre, ils permettront, peut-être, de stratifier les patients avec CHC suivant leur métagénome, ou encore de dégager des profils métagénomiques «particuliers » à risque, qui pourraient alors bénéficier d'un traitement modulateur de la microflore intestinale de type antibiotique, prébiotique ou probiotique. $\diamond$

Crosstallk between the liver and the gut: role of the microbiota in liver carcinogenesis

\section{LIENS D'INTÉRÊT}

Les auteurs déclarent n'avoir aucun lien d'intérêt concernant les données publiées dans cet article.

\section{RÉFÉRENCES}

1. Mantovani A, Allavena P, Sica A, Balkwill F. Cancerrelated inflammation. Nature 2008 ; 454 : 436-44.

2. Riordan $S M$, Williams R. The intestinal flora and bacterial infection in cirrhosis. J Hepatol 2006 ; 45 : 744-57.

3. Yu LX, Yan HX, Liu $Q$, et al. Endotoxin accumulation prevents carcinogen-induced apoptosis and promotes liver tumorigenesis in rodents. Hepatology 2010 ; 52 : 1322-33.

4. Dapito DH, Mencin A, Gwak Gy, et al. Promotion of hepatocellular carcinoma by the intestinal microbiota and TLR4. Cancer Cell $2012 ; 21: 504-16$.

5. Wang Z, Yan J, Lin H, et al. Toll-like receptor 4 activity protects against hepatocellular tumorigenesis and progression by regulating expression of DNA repair protein Ku70 in mice. Hepatology 2013 ; 57 : 1869-81.

6. Zhang $H L, Y_{u} L X, Y_{a n g} W$, et al. Profound impact of gut homeostasis on chemically induced pro-tumorigenic inflammation and hepatocarcinogenesis in rats.J Hepatol $2012 ; 57: 803-12$.

7. McKnite AM, Perez-Munoz ME, Lu L, et al. Murine gut microbiota is defined by host genetics and modulates variation of metabolic traits. PLoS One $2012 ; 7$ : e39191.

8. Friswell MK, Gika H, Stratford IJ, et al. Site and strainspecific variation in gut microbiota profiles and metabolism in experimental mice. PLoS One $2010 ; 5$ : e8584.

9. Agundez JA, Garcia-Martin E, Devesa MJ, et al. Polymorphism of the TLR4 gene reduces the risk of hepatitis $C$ virus-induced hepatocellular carcinoma. Oncology $2012 ; 82$ : 35-40.

10. Liang B, Chen R, Wang T, et al. Myeloid differentiation factor 88 promotes growth and metastasis of human hepatocellular carcinoma. Clin Cancer Res 2013; 19 : 2905-16.

11. Burcelin R, Chabo C, Blasco-Baque V, et al. Le microbiote intestinal à l'origine de nouvelles perspectives thérapeutiques pour les maladies métaboliques? Med Sci (Paris) 2013 ; 29 : 800-6.

12. Hoarau G, Colombel JF, Poulain D, Sendid B. Implication de la flore fongique intestinale dans le développement de la maladie de Crohn. Med Sci (Paris) $2013 ; 29: 691-3$.

13. Imler JL, Ferrandon D. Le printemps de l'immunité innée couronné à Stockholm. Med Sci (Paris) 2011 ; 27 : 1019-24.

14. Jamilloux $Y$, Henry T. Les inflammasomes : platesformes de l'immunité innée. Med Sci (Paris) 2013 ; $29: 975-84$. 